\title{
Network Simulation of Laminar Convective Heat and Mass Transfer over a Vertical Slender Cylinder with Uniform Surface Heat and Mass Flux
}

\author{
J. Zueco ${ }^{1 \dagger}$, O.A. Bég ${ }^{2}$, H.S. Takhar ${ }^{3}$ and G. Nath ${ }^{4}$ \\ ${ }^{I}$ Department Thermal Engineering and Fluids, Technical University of Cartagena, Cartagena, Murcia, 30202, Spain \\ ${ }^{2}$ Department Engineering and Mathematics, Sheffield Hallam University, Sheffield, S1 1WB, England, UK \\ ${ }^{3}$ Visiting Professor of Thermofluid Dynamics, 75 The Avenue, Sale, Manchester, M33 4GA, England, UK \\ ${ }^{4}$ KNIT Campus, Kamla Nehru Institute of Technology, Sultanpur, 228118, U.P., India
}

†Corresponding Author Email: joaquin.zueco@upct.es

(Received December 11, 2008; accepted May 5, 2010)

\begin{abstract}
The steady, laminar axisymmetric convective heat and mass transfer in boundary layer flow over a vertical thin cylindrical configuration in the presence of significant surface heat and mass flux is studied theoretically and numerically. The governing boundary-layer equations for momentum, energy and species conservation are transformed from a set of partial differential equations in a $(x, r)$ coordinate system to a $(\xi, \eta)$ system using a group of similarity transformations. The resulting equations are solved using the Network Simulation Method (NSM) for the buoyancy-assisted pure free convection and also the pure forced convection cases, wherein the effects of Schmidt number, Prandtl number and surface mass parameter on velocity, temperature and concentration distributions in the regime are presented graphically and discussed. For the buoyancy-assisted pure free convection case, nondimensional velocity $(\partial \mathrm{f} / \partial \eta)$ is found to increase with a rise in surface mass transfer $(\mathrm{S})$ but decrease with increasing Prandtl number $(\operatorname{Pr})$, particularly in the vicinity of the cylinder surface (small radial coordinate, $\eta$ ). Dimensionless temperature $(\theta)$ decreases however with increasing $S$ values from the cylinder surface into the free stream; with increasing Prandtl number, temperature is strongly reduced, with the most significant decrease at the cylinder surface. Dimensionless concentration $(\phi)$ is decreased continuously throughout the boundary layer regime with an increase in $\mathrm{S}$; conversely $\phi$ is enhanced for all radial coordinate values with an increase in Prandtl number. For the pure forced convection case, velocity increases both with dimensionless axial coordinate $(\xi)$ and dimensionless radial coordinate $(\eta)$ but decays smoothly with increasing Prandtl number and increasing Schmidt number, from the cylinder surface to the edge of the boundary layer domain. The model finds applications in industrial metallurgical processes, thermal energy systems, polymer processing, etc.
\end{abstract}

Keywords: Network simulation, Free and forced convection, Prandtl number, Surface mass parameter, Schmidt number, Energy systems.

\section{NOMENCLATURE}

\begin{tabular}{|c|c|}
\hline$a_{1}$ & function of $\lambda$ \\
\hline$a_{2}$ & function of $\lambda$ \\
\hline$a_{3}$ & function of $\lambda$ \\
\hline$a_{4}$ & function of $\xi$ \\
\hline$a_{5}$ & function of $\lambda$ \\
\hline$B^{*}$ & $\begin{array}{l}\text { flux constant }\left(\mathrm{m}^{2} \mathrm{~s}^{2} \text { Celsius) arising in }\right. \\
\text { definition of } S \text { in eqn. (8) }\end{array}$ \\
\hline$C$ & $\begin{array}{l}\text { species concentration in the boundary } \\
\text { layer }\end{array}$ \\
\hline$C_{\infty}$ & concentration at the free stream \\
\hline$D$ & species (mass) diffusivity \\
\hline$f$ & dimensionless stream function \\
\hline$g$ & acceleration due to gravity \\
\hline
\end{tabular}

$\begin{array}{ll}r_{o} & \text { radius of slender cylinder } \\ R e_{o} & \text { Reynolds number at cylinder surface } \\ R e_{x} & \text { local Reynolds number } \\ S & \text { dimensionless surface mass parameter } \\ S c & \text { Schmidt number } \\ T & \text { fluid temperature } \\ T_{\infty} & \text { free stream temperature } \\ u & \text { x-direction (axial) fluid velocity } \\ u_{\infty} & \text { free stream velocity } \\ v & \text { r-direction (radial) fluid velocity } \\ x & \text { axial coordinate, directed vertically } \\ & \text { upwards along cylinder longitudinal axis } \\ \alpha & \text { thermal diffusivity }\end{array}$




$\begin{array}{ll}G r_{o}{ }^{*} & \begin{array}{l}\text { Grashof number at cylinder surface } \\ \text { local Grashof number (buoyancy } \\ \text { parameter) }\end{array} \\ k & \begin{array}{l}\text { thermal conductivity of the fluid } \\ k\end{array} \\ m_{w} & \text { mass flux } \\ P r & \text { Prandtl number } \\ q_{w} & \text { heat flux } \\ r & \text { radial coordinate }\end{array}$

\section{INTRODUCTION}

Combined heat and mass transfer flows are of considerable importance in many applications. These include atmospheric and oceanic circulation, transport phenomena in power transformer electronics, heating and cooling processes in semi-conductor electronics, adsorption reactors, geophysical porous media, binary diffusion systems solar energy systems and polymer processing in the plastics industry. Mixed convection also is a very commonly encountered phenomenon and describes situations where both buoyancy and pressure forces exert an influence on the flow domain. Many applications exist in bio-membrane dynamics, surface science and bio-chemical engineering and the reader is referred to the monograph by Deen (1998). Many researchers have investigated coupled heat and mass transfer flows, often employing boundary layer theory to simplify the transport equations in order to yield numerical or closed-form solutions. An early study of laminar heat and mass transfer was conducted by Brown (1960). Tien and Campbell (1963) examined the combined boundary layer heat and mass transfer from spinning conical bodies. With regard to thermal convections flows within and external to cylindrical geometries many interesting studies have been communicated. Unsteady free convection from both plane surface and cylinder surfaces was analyzed by Goldstein and Briggs (1964). Evans et al. (1968) studied both theoretically and experimentally the internal unsteady free convection in a vertical cylinder where the core fluid temperature varied in the vertical direction. Vincent and Coeuret (1972) studied the external boundary layer heat and species transfer over a circular cylinder. Tripathi et al. (1973) presented an integral equation formulation for the transient heat and mass transfer in an infinite cylinder, with approximate solutions valid for small time values. Chen and Yuh (1980) studied the effects of buoyancy on free convection thermal and concentration boundary layers over a vertical cylinder showing that the local wall shear stress, local Nusselt number, and local Sherwood number increase with increasing curvature of the cylinder and that the local Sherwood number is boosted with increasing thermal buoyancy force increases. Merkin and Pop (1988) used Blasius and Görtler-type series expansions and also a finite difference method to study the natural convection boundary-layer flow on a horizontal circular cylinder with prescribed surface heat flux, showing that the Blasius solutions are more accurate in determining the temperature distributions and the Görtler-type expansions more successful in computing velocity distributions. Hossain and Nakayama (1993) analyzed computationally the surface mass flux and inertial porous medium effects on natural convection boundary layer flow over a vertical cylinder

$\begin{array}{ll}\beta & \text { coefficient of thermal expansion } \\ \delta & \text { boundary layer thickness } \\ v & \text { kinematic viscosity } \\ \psi & \text { dimensionless stream function parameter } \\ \xi & \text { dimensionless axial coordinate } \\ \eta & \text { dimensionless radial coordinate } \\ \Omega_{o} & \text { ratio of buoyancy to inertial forces } \\ \lambda & \text { function of the parameter, } \Omega_{0}\end{array}$

in a non-Darcian regime. Mamyoda and Asano (1993) used a numerical approach to present new correlations for the influence of diffusive surface mass suction/injection on conjugate heat and mass transfer over a cylinder for Reynolds numbers ranging from 10 to 100 , Prandtl or Schmidt numbers from 0.5 to 1.0 and surface mass suction or injection ratios from -0.2 to 0.1 . Takhar et al. (2000) analyzed the mixed convection flow over a continuous moving vertical slender cylinder with combined thermal and mass diffusion buoyancy effects using a perturbation method with Shanks transformation and the Blottner implicit difference method. They showed that a substantial overshoot in velocity is caused by an increase in buoyancy forces which also enhances surface skin friction, heat and mass transfer. Takhar et al. (2002) later considered the free convection boundary layer flow on an isothermal vertical thin cylinder embedded in a thermally-stratified high porosity non-Darcian medium showing that skin friction and heat transfer decrease with increasing ambient stratification, curvature and inertia parameters, but are elevated with a rise in the permeability parameter. Kumari and Nath (2004) used a numerical method to elucidate the influence of localized cooling/heating and injection/suction on the mixed convection boundary layer flow on a thin vertical cylindrical body, showing that heat transfer is affected considerably but not skin friction. They also found that the both curvature and buoyancy-assisted flow increase the skin friction and heat transfer. More recently Juncu (2005) has analyzed the combined heat and mass transfer from a circular cylinder in the presence of an internal heat/mass source. Very recently Zueco et al. (2009) have presented numerical solutions for the conjugate convective heat and mass transfer over a horizontal cylinder to a non-Darcian saturated porous medium. For coupled problems (involving buoyancy), the differential equations are more complex to solve and numerical methods are the only practicable means to yield solutions of interest to the engineering science community. In the present paper we shall investigate the axisymmetric mixed convective heat and mass transfer boundary layer flow of a viscous, incompressible Newtonian fluid over a slender vertical cylinder with uniform heat and mass flux. We utilize the Network Simulation Method solution for both the free and forced convection regimes. The present problem with variable surface mass flux has not been considered thus far in the scientific literature, despite important applications in polymer coating processes, energy systems, chemical reactor mixing and also industrial materials processing.

\section{GENERAL GUIDELINES}

The regime to be studied is illustrated below in Fig. 1a. 
The governing boundary-layer equations for the steady, axisymmetric, thermal convection heat and mass transfer along the cylinder external surface under buoyancy effects can be posed as follows, under the Boussinesq assumption, with reference to a cylindrical coordinate system $(\mathrm{x}, \mathrm{r})$ as follows:

\section{Conservation of Mass:}

$\frac{\partial(r u)}{\partial x}+\frac{\partial(r v)}{\partial r}=0$

Momentum :

$u \frac{\partial u}{\partial x}+v \frac{\partial u}{\partial r}= \pm g \beta\left(T-T_{\infty}\right) \pm$

$\pm g \beta\left(C-C_{\infty}\right)+\frac{v}{r} \frac{\partial}{\partial r}\left(r \frac{\partial u}{\partial r}\right)$

Thermal Energy (Heat):

$u \frac{\partial T}{\partial x}+v \frac{\partial T}{\partial r}=\frac{\alpha}{r} \frac{\partial}{\partial r}\left(r \frac{\partial T}{\partial r}\right)$

Species (Diffusion):

$u \frac{\partial C}{\partial x}+v \frac{\partial C}{\partial r}=\frac{D}{r} \frac{\partial}{\partial r}\left(r \frac{\partial C}{\partial r}\right)$

We neglect viscous dissipation in the energy Eq. (2) and assume that the concentration of the diffusing species in the binary mixture is significantly lower than the other chemical species present. As such the interfacial velocity at the cylinder surface due to mass diffusion is ignored. All parameters are defined in the nomenclature. We note that the first term on the right hand side of Eq. (2) is the buoyancy term due to temperature differences, and the second term is the buoyancy force due to concentration differences. For the case of a positive coefficient these buoyancy forces assist the upward flow; for the negative case they oppose the upward flow. The corresponding boundary conditions on the surface of the cylinder (for which $r=$ $r_{0}$ ) and far away from the surface $(\mathrm{r} \rightarrow \infty)$ take the form:

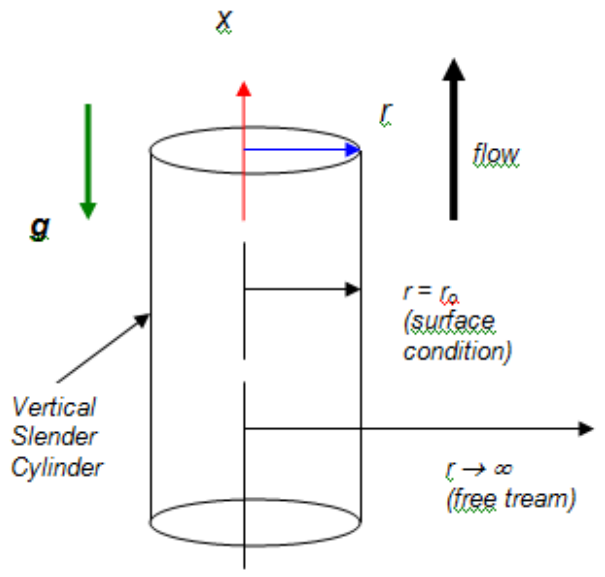

Fig. 1a. Physical model and coordinate system for convection over a vertical slender cylinder.

\subsection{Boundary conditions}

$$
\begin{aligned}
& u\left(x, r_{0}\right)=v\left(x, r_{0}\right)=0, \\
& \frac{\partial T\left(x, r_{0}\right)}{\partial r}=-\frac{q_{w}}{k}, \frac{\partial C\left(x, r_{0}\right)}{\partial r}=-\frac{m_{w}}{k} \\
& u(x, \infty)=u_{\infty}, T(x, \infty)=T_{\infty}, C(x, \infty)=C_{\infty} \\
& u(0, r)=u_{\infty}, T(0, r)=T_{\infty}, \\
& C(0, r)=C_{\infty}, r>r_{O}
\end{aligned}
$$

Introducing the following transformations:

$$
\begin{aligned}
& \psi=v r_{O} \frac{x}{\delta} f(\xi, \eta), \delta=\frac{x}{\left[\operatorname{Re}_{x}^{1 / 2}+G r_{x}^{1 / 5}\right]}, \\
& \theta=\frac{T-T_{\infty}}{\left[q_{w} \frac{\delta}{k}\right]}, \varphi=\frac{C-C_{\infty}}{\left[m_{w} \frac{\delta}{k}\right]}, r v=-\frac{\partial \psi}{\partial x}, \\
& \xi=\left[\frac{x}{r_{O} \operatorname{Re}_{o}}\right]^{1 / 4}, \operatorname{Re}_{O}=\frac{u_{\infty} r_{O}}{v}, \eta=\frac{r^{2}-r_{O}{ }^{2}}{2 r_{O} \delta_{O}}, \\
& \lambda=\left[\frac{1}{1+\Omega_{O} \xi^{6 / 5}}\right], \Omega_{O}=\left[\frac{G r_{O}}{\operatorname{Re}_{o}}\right]^{1 / 5}, \\
& G r_{O}^{*}=\frac{g \beta q_{w} r_{O}^{4}}{k v^{2}}, S=\frac{B * m_{w}}{\beta q_{w}}
\end{aligned}
$$

the conservations coordinates (1) to (4) reduce from an $(x, r)$ coordinate system to a $(\xi, \eta)$ coordinate system, with the continuity equation identically satisfied, to yield:

\section{Conservation of Momentum:}

$$
\begin{aligned}
& \left(1+2 \lambda \xi^{2} \eta\right) \frac{\partial^{3} f}{\partial \eta^{3}}+\left(2 \lambda \xi^{2}+a_{1} f\right) \frac{\partial^{2} f}{\partial \eta^{2}}+ \\
& a_{2}\left(\frac{\partial f}{\partial \eta}\right)^{2}+a_{3}(\theta+S \varphi)= \\
& =a_{4}\left(\frac{\partial^{2} f}{\partial \eta^{2}} \frac{\partial f}{\partial \xi}-\frac{\partial f}{\partial \eta} \frac{\partial^{2} f}{\partial \xi \partial \eta}\right)
\end{aligned}
$$

\section{Conservation of Energy:}

$$
\begin{aligned}
& \left(1+2 \lambda \xi^{2} \eta\right) \frac{\partial^{2} \theta}{\partial \eta^{2}}+\left(2 \lambda \xi^{2}+a_{1} \operatorname{Pr} f\right) \frac{\partial \theta}{\partial \eta}+ \\
& +\operatorname{Pr} a_{5} \frac{\partial f}{\partial \eta} \theta=\operatorname{Pr} a_{4}\left(\frac{\partial \theta}{\partial \eta} \frac{\partial f}{\partial \xi}-\frac{\partial f}{\partial \eta} \frac{\partial \theta}{\partial \xi}\right)
\end{aligned}
$$

Conservation of Species:

$$
\begin{aligned}
& \left(1+2 \lambda \xi^{2} \eta\right) \frac{\partial^{2} \varphi}{\partial \eta^{2}}+\left(2 \lambda \xi^{2}+a_{1} S c f\right) \frac{\partial \varphi}{\partial \eta}+ \\
& +S c a_{5} \frac{\partial f}{\partial \eta} \varphi=S c a_{4}\left(\frac{\partial \varphi}{\partial \eta} \frac{\partial f}{\partial \xi}-\frac{\partial f}{\partial \eta} \frac{\partial \varphi}{\partial \xi}\right)
\end{aligned}
$$

where Pr is the Prandtl number and Sc is the Schmidt number, the latter defining the ratio of the momentum to the mass diffusivity. Sc provides a measure of the 
relative effectiveness of momentum and mass transport by diffusion in the hydrodynamic (velocity) and concentration (species) boundary layers. The coefficients $a_{1}, a_{2}, a_{3}, a_{4}$ and $a_{5}$ are defined as follows:

$a_{1}=\frac{8-3 \lambda}{10}$

$a_{2}=-\frac{3(1-\lambda)}{5}$

$a_{3}= \pm(1-\lambda)^{5}$

$a_{4}=-\frac{\xi}{4}$

$a_{5}=-\frac{(2+3 \lambda)}{10}$

and

$$
\lambda=\frac{1}{1+\Omega_{o} \xi^{6 / 5}} . \quad \text { The }
$$

corresponding

transformed boundary conditions are:

Initial:

$$
\begin{aligned}
& \eta=0: \quad f(\xi, 0)=\frac{\partial f}{\partial \xi}(\xi, 0)=0, \\
& \frac{\partial \theta}{\partial \xi}(\xi, 0)=\frac{\partial \phi}{\partial \xi}(\xi, 0)=-1
\end{aligned}
$$

End:

$$
\eta \rightarrow \infty: \frac{\partial f}{\partial \xi}(\xi, \infty)=\lambda^{2}, \theta(\xi, \infty)=\phi(\xi, \infty)=0
$$

\section{SPecial Flow CaSes}

A number of special cases of the general mathematical model defined by Eqs. (9) to (11) under conditions (17, 18) can now be considered.

\subsection{Case A: Pure Free Convection Regime}

By setting $\mathrm{u}_{\infty}, \quad \Omega_{\mathrm{o}} \rightarrow \infty, \quad \lambda \rightarrow 0 \quad$ and $\xi=\left[\frac{x}{r_{O} G r_{O} *}\right]^{1 / 10}$, we obtain the case for a free convection regime :

\section{Conservation of Momentum:}

$$
\begin{aligned}
& \frac{\partial^{3} f}{\partial \eta^{3}}+a_{1} f \frac{\partial^{2} f}{\partial \eta^{2}}+a_{2}\left(\frac{\partial f}{\partial \eta}\right)^{2}+a_{3}(\theta+S \varphi)= \\
& =a_{4}\left(\frac{\partial^{2} f}{\partial \eta^{2}} \frac{\partial f}{\partial \xi}-\frac{\partial f}{\partial \eta} \frac{\partial^{2} f}{\partial \xi \partial \eta}\right)
\end{aligned}
$$

\section{Conservation of Energy:}

$$
\begin{aligned}
& \frac{\partial^{2} \theta}{\partial \eta^{2}}+a_{1} \operatorname{Pr} f \frac{\partial \theta}{\partial \eta}+\operatorname{Pr} a_{5} \frac{\partial f}{\partial \eta} \theta= \\
& =\operatorname{Pr} a_{4}\left(\frac{\partial \theta}{\partial \eta} \frac{\partial f}{\partial \xi}-\frac{\partial f}{\partial \eta} \frac{\partial \theta}{\partial \xi}\right)
\end{aligned}
$$

\section{Conservation of Species:}

$$
\begin{aligned}
& \frac{\partial^{2} \varphi}{\partial \eta^{2}}+a_{1} S c f \frac{\partial \varphi}{\partial \eta}+S c a_{5} \frac{\partial f}{\partial \eta} \varphi= \\
& =S c a_{4}\left(\frac{\partial \varphi}{\partial \eta} \frac{\partial f}{\partial \xi}-\frac{\partial f}{\partial \eta} \frac{\partial \varphi}{\partial \xi}\right)
\end{aligned}
$$

Of course the coefficients in (13) to (16) reduce to: $a_{1}=$ $8 / 10, \mathrm{a}_{2}=-3 / 5, \mathrm{a}_{3}= \pm 1$ (positive for buoyancyassisted flows and negative for buoyancy-impeded flows), $a_{4}=-\xi / 10$ and $a_{5}=-2 / 10$ which simply are substituted into Eqs .(20) to (22).

\subsection{Case B: Pure Forced Convection Regime}

For this case the buoyancy forces in (9) vanish. We set $\Omega_{0} \rightarrow 0, \lambda \rightarrow 1$ and $\mathrm{Gr}_{\mathrm{x}}{ }^{*} \rightarrow 0$. The reduced flow equations for forced convection are therefore:

\section{Conservation of Momentum:}

$$
\begin{aligned}
& \left(1+2 \xi^{2} \eta\right) \frac{\partial^{3} f}{\partial \eta^{3}}+\left(2 \xi^{2}+a_{1} f\right) \frac{\partial^{2} f}{\partial \eta^{2}}= \\
& =a_{4}\left(\frac{\partial^{2} f}{\partial \eta^{2}} \frac{\partial f}{\partial \xi}-\frac{\partial f}{\partial \eta} \frac{\partial^{2} f}{\partial \xi \partial \eta}\right)
\end{aligned}
$$

\section{Conservation of Energy:}

$$
\begin{aligned}
& \left(1+2 \xi^{2} \eta\right) \frac{\partial^{2} \theta}{\partial \eta^{2}}+\left(2 \xi^{2} \eta+a_{1} \operatorname{Pr} f\right) \frac{\partial \theta}{\partial \eta}+ \\
& +\operatorname{Pr} a_{5} \frac{\partial f}{\partial \eta} \theta=\operatorname{Pr} a_{4}\left(\frac{\partial \theta}{\partial \eta} \frac{\partial f}{\partial \xi}-\frac{\partial f}{\partial \eta} \frac{\partial \theta}{\partial \xi}\right)
\end{aligned}
$$

\section{Conservation of Species:}

$$
\begin{aligned}
& \left(1+2 \xi^{2} \eta\right) \frac{\partial^{2} \varphi}{\partial \eta^{2}}+\left(2 \xi^{2} \eta+a_{1} S c f\right) \frac{\partial \varphi}{\partial \eta}+ \\
& +S c a_{5} \frac{\partial f}{\partial \eta} \varphi=S c a_{4}\left(\frac{\partial \varphi}{\partial \eta} \frac{\partial f}{\partial \xi}-\frac{\partial f}{\partial \eta} \frac{\partial \varphi}{\partial \xi}\right)
\end{aligned}
$$

Again the coefficients in (13) to (16) will simplify in this case to: $a_{1}=5 / 10, a_{2}=0, a_{3}=0, a_{4}=-\xi / 4$ and $a_{5}=$ $-5 / 10$ in Eqs. (23) to (25).

In the present study we analyze the variation of velocity $(\partial \mathrm{f} / \partial \eta)$, temperature $(\theta)$ and concentration $(\phi)$ distributions with axial $(\xi)$ and radial coordinate $(\eta)$, in the regime. Of course gradient functions of these quantities lead to more complex functions for the local frictional factor $\left(\mathrm{c}_{\mathrm{fx}}\right)$, Nusselt number $\left(\mathrm{Nu}_{\mathrm{x}}\right)$ and local Sherwood number $\left(\mathrm{Sh}_{\mathrm{x}}\right)$ respectively; these are excluded here for brevity.

\section{Numerical Solution}

Although solutions have been obtained for both free and forced convection cases discussed in section 3, we present for conservation of space, a specific numerical solution for only the free convection case, defined by Eqs. (20) to (22), which has boundary conditions (17) and (18), the latter being the same for the general flow model, as well as the forced convection case. In the present study we have considered only the buoyancy- 
assisted free convection case for which $\mathrm{a}_{3}=+1$ in Eq. (20). The Network Simulation Method (NSM) has been used solve the system of partial differential Eqs (20) to (22) under boundary conditions $(17,18)$. This method has applied to a wide range of heat transfer problems by Zueco and co-workers (Zueco et al. 2004; Zueco 2006; Zueco 2008). Further studies using NSM have been presented by Bég et al. (2008a, 2008b, 2009). In NSM a number of networks are connected in series to make up the whole medium and boundary conditions are added by means of special electrical devices (current or voltage control-sources). NSM is founded on the classical thermoelectric analogy between thermal and electrical variables. Nevertheless, its capacity to implement in the model any kind of non-linearity (due to boundary conditions, phase-change processes, temperature dependencies of the thermal properties, etc) distinguishes NSM from the analogies generally exposed in text books. The electrical analogy relates the electrical current $(J)$ with the velocity fluxes, heat fluxes and mass fluxes, while the electrical potential (V) is equivalent to velocities, temperatures and concentrations. We define the variable $\Phi=\frac{\partial f}{\partial \eta}$ to reduce the third order partial differential momentum Eq. (20) to a second order differential equation. The Eqs. (20) to (22) then reduce to:

For $\xi>0$ :

Conservation of momentum:

$$
\begin{aligned}
& \frac{\partial^{2} \Phi}{\partial \eta^{2}}+a_{1} f \frac{\partial \Phi}{\partial \eta}+a_{2} \Phi^{2}+a_{3}(\theta+S \varphi)= \\
& =a_{4}\left[\frac{\partial f}{\partial \xi} \frac{\partial \Phi}{\partial \eta}-\Phi \frac{\partial \Phi}{\partial \xi}\right]
\end{aligned}
$$

\section{Conservation of energy:}

$$
\begin{aligned}
& \frac{\partial^{2} \theta}{\partial \eta^{2}}+a_{1} \operatorname{Pr} f \frac{\partial \theta}{\partial \eta}+a_{5} \operatorname{Pr} \Phi \theta= \\
& =a_{4} \operatorname{Pr}\left[\frac{\partial f}{\partial \xi} \frac{\partial \theta}{\partial \eta}-\Phi \frac{\partial \theta}{\partial \xi}\right]
\end{aligned}
$$

\section{Conservation of species:}

$$
\begin{aligned}
& \frac{\partial^{2} \varphi}{\partial \eta^{2}}+a_{1} S c f \frac{\partial \varphi}{\partial \eta}+a_{5} S c \Phi \varphi= \\
& =a_{4} S c\left[\frac{\partial f}{\partial \xi} \frac{\partial \varphi}{\partial \eta}-\Phi \frac{\partial \varphi}{\partial \xi}\right]
\end{aligned}
$$

Figure $1 \mathrm{~b}$ shows the flow chart to obtain the numerical solution by means of the Network Simulation Method. In the NSM technique, discretization of the differential equations is founded on the difference-finite formulation, where only a discretization of the spatial co-ordinates is necessary, time remaining as a real continuous variable. The starting point is the set of finite-differential equations, one for each control volume, obtained by spatial discretization of the transformed Eqs. (26-28). Based on these equations, a network model (that incorporates the three equations) is designed. It is assumed that the electrical variable of voltage is equivalent to the variables f, $\theta$ and $\Phi$, while the current is equivalent to the fluxes $(\partial \mathrm{f} / \partial \eta, \partial \theta / \partial \eta$, $\partial \Phi / \partial \eta)$. The whole network model, including the devices associated with the boundary conditions, is solved by the numerical computer code Pspice (1994) and the graph solution can be obtained by means the Probe software of Pspice.

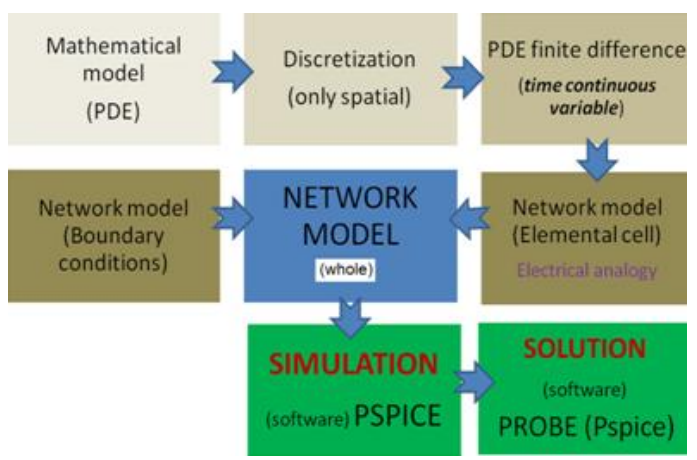

Fig. 1b. Flow chart of the Network Simulation Method.

A first-order central-difference approximation is used for the first derivate and a second-order central difference approximation is used for the second derivate. The computational domain is divided into meshes each of dimension $2 \Delta \xi$ and $2 \Delta \eta$ in $\xi$-space and $\eta$-space, respectively. The Fig. 1c shows the physical medium reticulation with elementals cells connected between them, where it is possible observed that the thickness of the elemental cell is $2 \Delta \eta$. This connecting requires, equal potentials at the contact points of adjoining cells and equal outward-inward transference flows in adjoining elements.

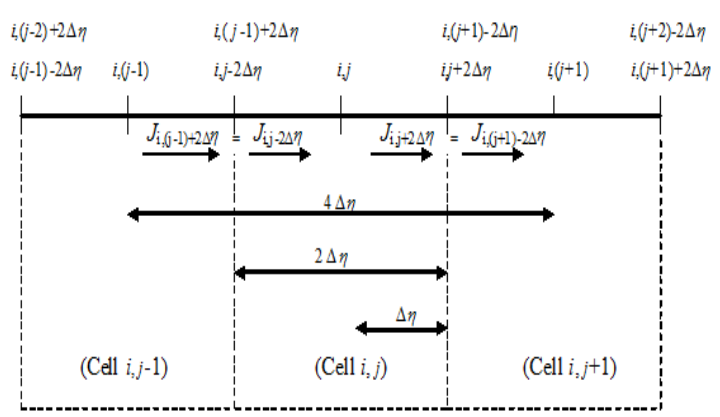

Fig. 1c. Mesh system used.

The discretized form of Eqs. (26) to (28) then become:

$$
\begin{aligned}
& \frac{\Phi_{i, j+1}+\Phi_{i, j-1}-2 \Phi_{i, j}}{\Delta \eta^{2}}+a_{1} f_{i, j} \frac{\Phi_{i, j+1}-\Phi_{i, j-1}}{2 \Delta \eta}+ \\
& a_{2} \Phi_{i, j}^{2}+a_{3}\left(\theta_{i, j}+S \varphi_{i, j}\right)=a_{4}\left[\frac{\left(f_{i+1, j}-f_{i-1, j}\right)}{2 \Delta \xi}\right. \\
& \left.\frac{\left(\Phi_{i, j+1}-\Phi_{i, j-1}\right)}{2 \Delta \eta}-\Phi_{i} \frac{\left(\Phi_{i+1, j}-\Phi_{i-1, j}\right)}{2 \Delta \xi}\right]
\end{aligned}
$$




$$
\begin{aligned}
& \frac{\theta_{i, j+1}+\theta_{i, j-1}-2 \theta_{i, j}}{\Delta \eta^{2}}+a_{1} \operatorname{Pr} f_{i, j} \frac{\left(\theta_{i, j+1}-\theta_{i, j-1}\right)}{2 \Delta \eta}+ \\
& a_{5} \operatorname{Pr} \Phi_{i, j} \theta_{i, j}=a_{4} \operatorname{Pr}\left[\frac{\left(f_{i+1, j}-f_{i-1, j}\right)}{2 \Delta \xi}\right. \\
& \left.\frac{\left(\theta_{i, j+1}-\theta_{i, j-1}\right)}{2 \Delta \eta}-\Phi_{i, j} \frac{\left(\theta_{i+1, j}-\theta_{i-1, j}\right)}{2 \Delta \xi}\right] \\
& \frac{\varphi_{i, j+1}+\varphi_{i, j-1}-2 \varphi_{i, j}}{\Delta \eta^{2}}+a_{1} S c f_{i, j} \frac{\left(\varphi_{i, j+1}-\varphi_{i, j-1}\right)}{2 \Delta \eta}+ \\
& +a_{5} S c \Phi_{i, j} \varphi_{i, j}=a_{4} S c\left[\frac{\left(f_{i+1, j}-f_{i-1, j}\right)}{2 \Delta \xi}\right. \\
& \left.\frac{\left(\varphi_{i, j+1}-\varphi_{i, j-1}\right)}{2 \Delta \eta}-\Phi_{i, j} \frac{\left(\varphi_{i+1, j}-\varphi_{i-1, j}\right)}{2 \Delta \xi}\right]
\end{aligned}
$$

In the Eqs. (29-31) all the terms can be treated as a current. Therefore the discretized momentum Eq. (29) the following currents are defined:

$$
\begin{aligned}
& J_{\Phi, i, j-1}=\frac{\Phi_{i, j-1}-\Phi_{i, j}}{\Delta \eta} \\
& J_{\Phi, i, j+1}=\frac{\Phi_{i, j}-\Phi_{i, j+1}}{\Delta \eta} \\
& J_{\Phi, a 1, i, j}=\frac{a_{1} f_{i, j}\left(\Phi_{i, j+1}-\Phi_{i, j-1}\right)}{2} \\
& J_{\Phi, a 2, i, j}=\Delta \eta a_{2} \Phi_{i, j}^{2} \\
& J_{\Phi, a 3, i, j}=\Delta \eta a_{3}\left(\theta_{i, j}+S \varphi_{i, j}\right) \\
& J_{\Phi, a 4, i, j}=a_{4} \frac{\left(f_{i+1, j}-f_{i-1, j}\right)\left(\Phi_{i, j+1}-\Phi_{i, j-1}\right)}{4 \Delta \xi} \\
& -a_{4} \Phi_{i} \Delta \eta \frac{\left(\Phi_{i+1, j}-\Phi_{i-1, j}\right)}{2 \Delta \xi}
\end{aligned}
$$

Implementing Kirchhoff's law for electrical currents from circuit theory, the network model is then obtained.

$$
\begin{aligned}
& J_{\Phi, i, j-1}-J_{\Phi, i, j-1}+J_{\Phi, a 1, i, j}+J_{\Phi, a 2, i, j}+ \\
& +J_{\Phi, a 3, i, j}-J_{\Phi, a 4, i, j}=0
\end{aligned}
$$

The modeling is realized by means of two resistors of value " $\Delta \eta$ " ( $\left.J_{\Phi, i, j-1} ; J_{\Phi, i, j-1}\right)$ and other currents are implemented with voltage control current generator. To introduce the boundary conditions, voltage and current sources and employed. Similarly, the discretized energy Eq. (30) can be modeled by means of the NSM. For both equations, initially it is necessary to solve the 1-D problem $\quad\left(\xi=0\right.$, with $J_{\Phi, a 4, i, j}=0 \quad$ ), this initial numerical solution serves as the input boundary condition for a solution for $\xi>0$, which is twodimensional. To obtain a symmetric network model the computational domain (Fig. 2) is divided by two to form meshes of dimension $\Delta \xi=\mathrm{L}_{\xi} / \mathrm{N}_{\xi}$ and $\Delta \eta=\mathrm{L}_{\eta} / \mathrm{N}_{\eta}$ in $\xi$-space and $\eta$-space, respectively. As such the new value of the resistors of each cell is " $\Delta \eta / 2$ ". After experimenting with a few sets of mesh sizes, a region of integration of $\mathrm{N}_{\xi}=40 \mathrm{xN}_{\eta}=150$ cells has been employed, with $L_{\xi}=1.0$ and $L_{\eta}=15.0$. Once the complete network model is designed, for which few programming rules are needed since not many devices form the network, a computer Pspice (1994) is used for a PC-based simulation yielding the full numerical solution.

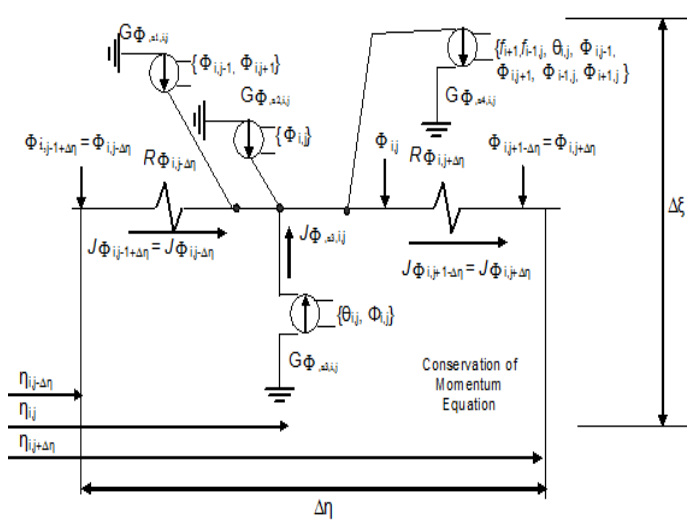

Fig. 2. Network model of the elemental cell for the momentum Eq. (20).

\section{RESUlts AND Discussion}

Selected graphical results are presented in Figs. 3 to 11. The regime is governed for the free convection case (figures 3 to 8 ) by the parameters S, Pr, Sc. As we have stated earlier the coefficient, $\mathrm{a}_{3}=+1$, in Eqs. (20) to (22) corresponding to buoyancy-assisted flow.

Selected graphical results are presented in Figs. 3 to 11. The regime is governed for the free convection case (Figs 3 to 8) by the parameters S, Pr, Sc. As we have stated earlier the coefficient, $\mathrm{a}_{3}=+1$, in Eqs. (20) to (22) corresponding to buoyancy-assisted flow. The graphs (9) to (11) correspond to the forced convection case and are independent of the $S$ parameter. In figures 3 to 8 we have used $\mathrm{Pr}=0.7, \mathrm{Sc}=0.7$ and $\mathrm{S}=0.5$ as default values, which correspond to for example hydrogen gas diffusing in air (Incropera 1996)

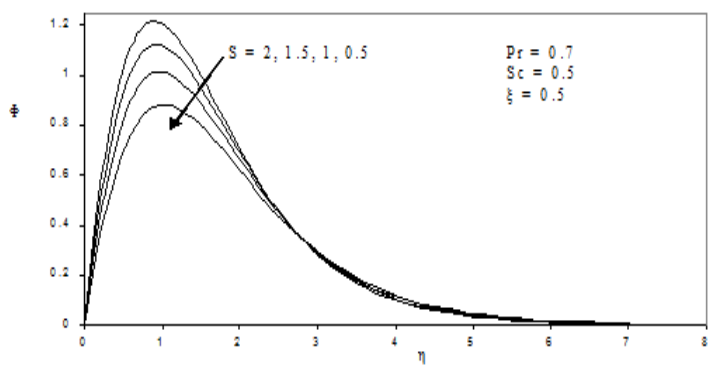

Fig. 3. Dimensionless velocity variation $(\Phi)$ with radial coordinate $(\eta)$ for various surface mass parameters $(S)$ (pure free convection). 
In Fig. 3 we observe a significant increase in the dimensionless velocity, $\Phi$, with rising surface mass transfer parameter, $S$. This is especially pronounced in the proximity of the cylinder surface. Profiles ascend from zero at the wall, peak close to the wall and decay asymptotically to zero in the free stream, far from the cylinder surface. Increasing surface mass transfer therefore serves to accelerate the boundary layer flow nearer to the wall but is much less effective further from the cylinder surface.

Clearly $S=\frac{B^{*} m_{w}}{\beta q_{w}}$ is directly proportional to the mass flux (mw). The parameter S couples the momentum Eq. (9) with the species diffusion Eq. (11) in the term, $S \phi$. This is effectively a species buoyancy force. Increased species buoyancy will aid the momentum development and this will act to accelerate the flow. Since species concentration is maximized at the cylinder surface it is logical to expect that species buoyancy forces will also be most vigorous in this zone, explaining the strong rise in velocity near the cylinder surface in Fig. 3. Further from the cylinder surface we observe almost negligible effect of the species buoyancy force, which agrees with the physical interpretation we have made.

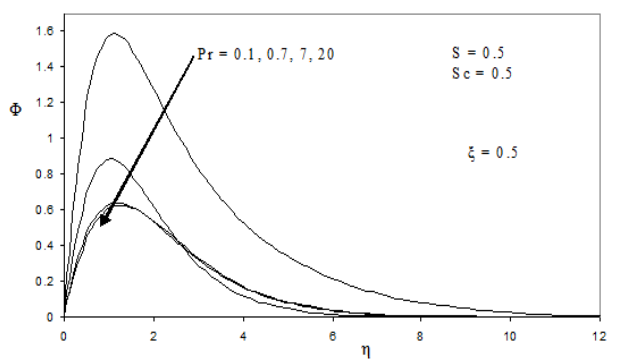

Fig. 4. Dimensionless velocity variation $(\Phi)$ with radial coordinate $(\eta)$ for various Prandtl numbers $(\operatorname{Pr})$ (pure free convection).

In Fig. 4, an increase in Prandtl number, $P r$, for the buoyancy-assisted free convection regime, causes a strong decrease in the velocity, $\Phi$, again closer to the cylinder surface. Peak velocity decreases from 1.58 approximately for $P r=0.1$ to about 0.6 for $P r=20$. Increasing Prandtl number implies an increase in dynamic viscosity and a decrease in thermal conductivity of the fluid. As such lower Prandtl numbers correspond to gases (low viscosity and high thermal conductivity) and higher Prandtl numbers to oils (high viscosity, lower thermal conductivities), (Incropera 1994). For low Prandtl number the fluid will flow faster i.e. velocities will be increased. Prandtl number simulates the ratio of momentum diffusivity to thermal diffusivity. Higher Prandtl numbers correspond to a thinner thermal boundary layer thickness and more uniform temperature distributions across the boundary layer. Hence the thermal boundary layer will be much less in thickness than the hydrodynamic (translational velocity) boundary layer or concentration (species) boundary layer. Smaller $P r$ fluids have higher thermal conductivities so that heat can diffuse away from the vertical surface faster than for higher $P r$ fluids (thicker boundary layers).
Figure 5 shows that for the free convection case, temperature $(\theta)$ decreases continuously through the boundary layer regime, normal to the cylinder surface, with an increase in surface mass transfer parameter, $S$. At the cylinder surface where the temperature in all cases is a maximum, values fall from 1.8 for $S=0.5$ to 1.54 for the maximum value of $\mathrm{S}$ of 2 . Again the computations in Fig. 5 correspond to hydrogen gas diffusing in air $(\mathrm{Pr}=0.7, \mathrm{Sc}=0.5)$. The temperature (energy) Eq. (10) is coupled to the momentum Eq. (9) via the thermal and species buoyancy term, $+a_{3}(\theta+S \phi)$. The latter term couples the energy Eq. (10) to the species conservation Eq. (11). Furthermore the parameters, $a_{1}=\frac{8-3 \lambda}{10}, a_{4}=-\frac{\xi}{4}, a_{5}=-\frac{(2+3 \lambda)}{10}$ all arise in the species Eq. (11). These parameters all feature the parameter, $\lambda=\frac{1}{1+\Omega_{o} \xi^{6 / 5}}$ which contains the parameter $\Omega_{O}=\left[\frac{G r_{O}^{*}}{\operatorname{Re}_{O}}\right]^{1 / 5}$ this clearly featuring the free convection Grashof number, $G r_{O}^{*}=\frac{g \beta q_{w_{O}}{ }^{4}}{k v^{2}}$.

As such there is a very strong interplay between the energy (heat) diffusion and the species diffusion. Restricting attention for the moment to the $S$ parameter, as in the case of the velocity field, $S$ is directly proportional to mass flux, which, in accordance with the boundary conditions, is maximized at the cylinder surface. However this parameter will serve to directly boost species buoyancy which will primarily drive the momentum boundary layer. To counterbalance the aiding contribution of species buoyancy to flow, there must arise an opposing effect to thermal diffusion. As such the parameter, $S$, will effectively oppose heat diffusion and will act to cool the boundary layer regime, as testified to by the concurrent decrease in $\theta$ values, which we observe in Fig. 5 are pronounced principally in the near-wall region.

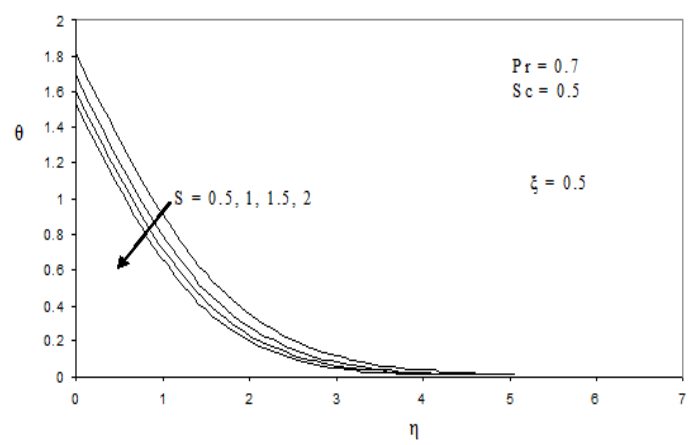

Fig. 5. Dimensionless temperature variation $(\theta)$ with radial coordinate $(\eta)$ for various surface mass parameters (S) (pure free convection)

Further from the cylinder surface since $S$ exerts a progressively decreasing effect, it will tend to influence temperature fields minimally. These results concur with earlier studies by Merkin and Pop (1987) who also found, as we have, that free convection (buoyancy) 
effects exert a diminished role far from the cylinder surface and also further along the cylinder surface from the leading edge. A similar effect has been documented by Lira (2008).

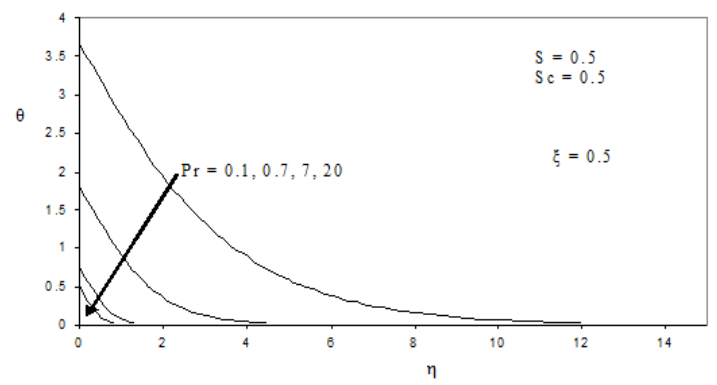

Fig. 6. Dimensionless temperature variation $(\theta)$ with radial coordinate $(\eta)$ for various Prandtl numbers $(\operatorname{Pr})$ (pure free convection).

With an increase in Prandtl number, $P r$, as shown in Fig. 6, temperatures are also decreased strongly throughout the boundary layer regime. Maximum temperature once again occurs for all $P r$ values at the cylinder surface $(\eta=0)$; profiles descend smoothly from the surface to zero far from the surface. As explained earlier, increasing Prandtl number, corresponds to a decrease in thermal conductivity of the primary fluid. For $\operatorname{Pr}=0.1$, thermal diffusivity will be ten times the momentum diffusivity; as such heat will diffuse ten times faster than momentum and this will enhance temperatures i.e. heat the boundary layer regime. Conversely at the opposite spectrum of Prandtl numbers studied in Fig. 6, i.e. $P r=2.0$, momentum diffusivity will be double the thermal diffusivity manifesting in viscous diffusion rate being twice the energy diffusion rate. Temperatures ill therefore be decreased considerably in the boundary layer, as indicated in Fig. 6. These results are significant in physical applications. To enhance temperature distributions in the boundary layer flow from a vertical cylinder, a regime which arises in for example condenser system design and materials processing, lower Prandtl number fluids will perform better. Air $(\mathrm{Pr}$ $=0.7)$ therefore is more advisable in such applications than water $(\operatorname{Pr}=7.0)$.

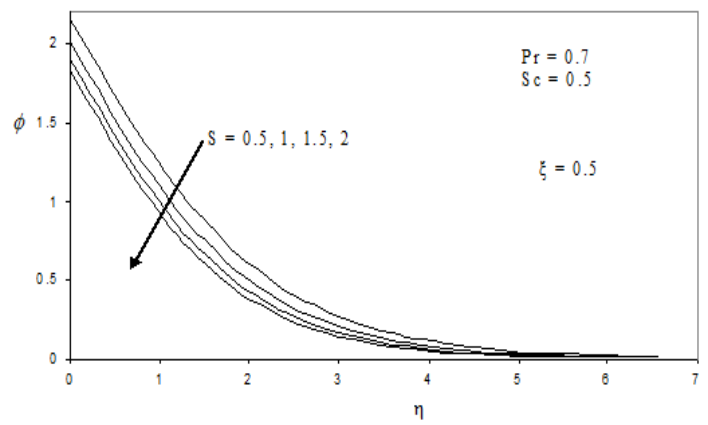

Fig. 7. Dimensionless concentration variation $(\phi)$ with radial coordinate $(\eta)$ for various surface mass parameters (S) (pure free convection).

Concentration distribution, $\phi$ versus $\eta$ is plotted for various surface mass transfer parameter $(S)$ values in Fig. 7. The $S$ parameter arises in the collective buoyancy term in equation (20), viz $+a_{3}(\theta+S \phi)$. Increasing $S$ serves to clearly reduce the concentration $(\phi)$ values in the flow regime; all profiles fall from a maximum at the surface to zero far from the cylinder. As indicated earlier, an increase in $S$ will significantly boost the species buoyancy force in the momentum Eq. (9)- however both energy and species diffusion will be curtailed by the increase in velocity fields. With greater mass flux $\left(m_{w}\right), S$ is increased- concentration along the cylinder surface is increased i.e. with the $\xi$-coordinate (streamwise). Concentration distribution is however reduced, across the boundary layer i.e. progressively less species is diffused from the cylinder surface to the free stream. The effects are most potent at the near-wall regime, and then depleted further from the cylinder, in a similar fashion to the velocity field and temperature field.

Conversely an increase in Prandtl number is found to increase concentration $(\phi)$ values in Fig. 8. An increase in $\mathrm{Pr}$ also implies greater heat transfer rates. A similar trend has been observed in the study by Ganesan and Rani (2000). Prandtl number signifies the ratio of viscous diffusion to thermal diffusion. With greater $\operatorname{Pr}$ values, viscous diffusion $>$ thermal diffusion. This is disadvantageous to the temperature field, but beneficial to the species diffusion field since greater momentum diffusion aids the advection of mass in the flow. In design applications, therefore to achieve a better distribution of species across the boundary layer (transverse to the cylinder surface), higher Prandtl number liquids will be more logical than lower Prandtl numbers.

The interplay of momentum, thermal and species diffusion will imply inevitably that all three variables cannot be simultaneously maximized, irrespective of the magnitude of the buoyancy forces involved. A strategic approach is therefore required in selecting primary fluids which posses high thermal diffusivitiies (low Prandtl numbers) for temperature enhancement or low thermal diffusivities (high Prandtl numbers) for species diffusion enhancement. Such aspects have been elucidated more rigorously with respect to experimental observations by Gebhart et al. (1988) who have considered salt diffusion in water from vertical cylinders under combined buoyancy forces and many other primary-binary diffusion flows.

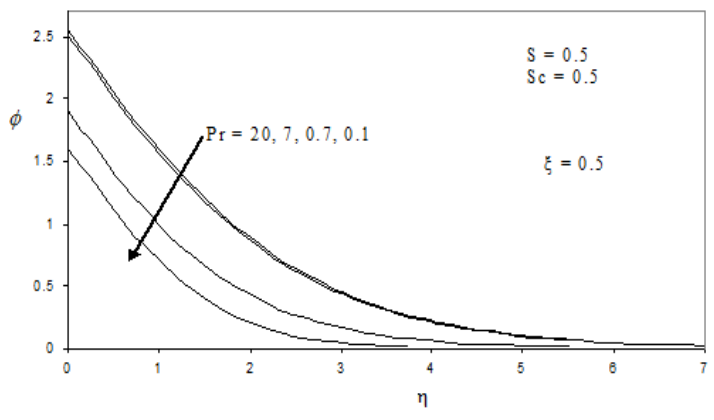

Fig. 8. Dimensionless concentration variation $(\phi)$ with radial coordinate $(\eta)$ for various Prandtl surface mass parameters (pure free convection). 


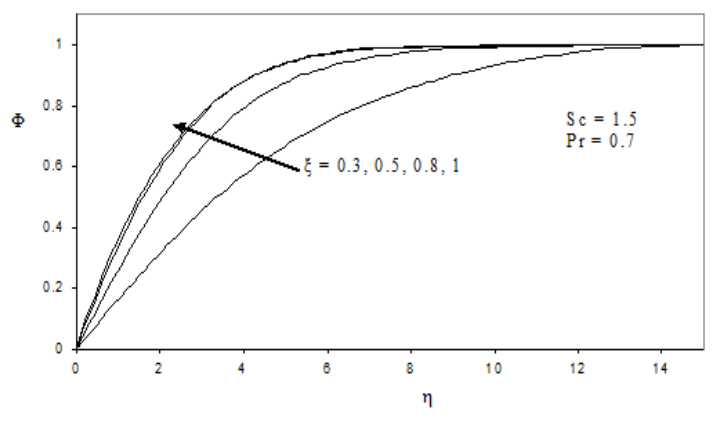

Fig. 9. Dimensionless velocity variation $(\Phi)$ with radial coordinate $(\eta)$ for various axial coordinate values $(\xi)$ (pure forced convection).

The influence of axial coordinate $(\xi)$ on the velocity distribution $(\Phi)$ through the boundary layer (i.e. with radial coordinate, $\eta$ ) is illustrated in Fig. 9, for the case of pure forced convection i.e. where buoyancy effects are neglected. With increasing distance upwards along the surface of the cylinder i.e. for increasing $\xi$, velocity clearly increases substantially. The Schmidt number (Sc) in Fig. 9 equals 1.5 implying that the momentum diffusivity exceeds the species diffusivity. Velocities all converge to the same value in the free stream i.e. unity, as prescribed by the boundary condition there. Merkin and Pop (1987) have also identified, using asymptotic methods, the acceleration of flow along the cylinder, from the leading edge $(\xi=0)$; they attribute this to the dominating role of forced convection further along the cylinder and further from the cylinder surface (in the $\eta$ direction), which agrees with our computations. Momentum development therefore is escalated with further distance from the leading edge. This is an important feature in industrial design, for example in the surface treatment of cylindrical systems, where greater velocities may be required to achieve improved efficiency. The absence of buoyancy forces in the case studied in Fig. 9, indicates that these will not be dominant factors controlling the acceleration of flow along the cylinder, although they will influence the flow transverse to the cylinder.

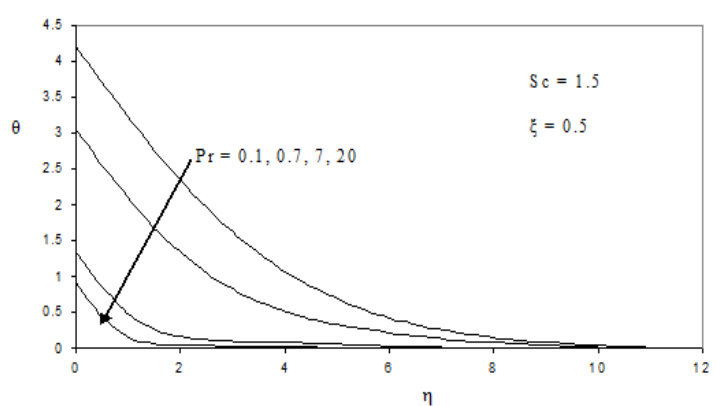

Fig. 10. Dimensionless temperature variation $(\theta)$ with radial coordinate $(\eta)$ for various Prandtl number values (Pr) (pure forced convection).

As with the pure free convection case (Fig 6), the temperature function $(\theta)$ values for the pure forced convection case, figure 10 , also decrease with increasing Prandtl number. Comparing with Fig 6 (pure free convection case) values are somewhat higher for the pure forced convection case, and it should be noted that the Sc value is 1.5 in Fig 10 compared with 0.5 in Fig. 6. Prandtl number is a thermophysical property at a given temperature and pressure. As such it is associated with actual liquids. It will exert a similar influence on the temperature distribution whether in the pure free convection or pure forced convection scenarios. With buoyancy present, temperatures will be affected adversely. Without buoyancy they will be higher.

Finally the influence of Schmidt number $(\mathrm{Sc})$ on the concentration distribution $(\phi)$ versus radial coordinate $(\eta)$ are shown in Fig. 11. For $\mathrm{Sc}<1$ the momentum diffusivity is lower than the species (mass) diffusivity and the species diffusion rate exceeds the momentum diffusion rate. For $\mathrm{Sc}>1$ this is reversed. Higher values of Sc correspond to higher density species diffusing in air e.g. $\mathrm{Sc}=1.0$ implies Methanol diffusing in air, $\mathrm{Sc}=$ 2.0 implies Ethylbenzene diffusing in air. Increasing Sc lowers the chemical molecular diffusivity of the species which allows easier diffusion. Increasing Schmidt number clearly reduces the concentration values throughout the regime. Peak values (i.e. at the cylinder surface, $\eta=0$ ) plunge from 1.8 for $\mathrm{Sc}=0.5$ to 0.5 for $\mathrm{Sc}=2$. For higher Schmidt numbers, therefore species diffusion is inhibited in the boundary layer regime.

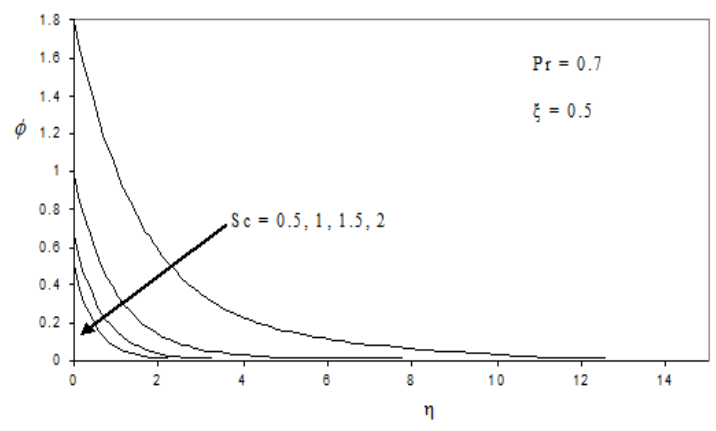

Fig. 11. Dimensionless concentration variation $(\phi)$ with radial coordinate $(\eta)$ for various Schmidt numbers $(\mathrm{Sc})$ (pure forced convection).

\section{Conclusion}

A mathematical model has been developed for the simultaneous heat, mass and momentum transfer in axisymmetric incompressible boundary layer flow past a vertical slender cylindrical body with surface heat and mass flux. The governing boundary layer equations have been transformed from an (x,r) coordinate system to a $(\xi, \eta)$ system. Two special cases of the flow model have been discussed, namely pure free convection and also pure forced convection. A numerical solution based on the well-tested Network Simulation Method (NSM) based on thermo-electrical analogies has been presented for both cases. Our computations have shown that for the pure free convection case, buoyancyassisted pure free convection case, increasing surface mass transfer parameter ( $\mathrm{S}$ ) boosts velocity but lowers temperature $(\theta)$ and concentration function $(\phi)$. Increasing Prandtl number (Pr), however decreases both velocity $(\Phi=\partial \mathrm{f} / \partial \eta)$ and temperature $(\theta)$ but increases 
concentration values in the boundary-layer regime for the pure free convection case. For the pure forced convection case, temperature $(\theta)$ is also reduced with an increase in Prandtl number and also Schmidt number (Sc); concentration is however increased for all values of radial coordinate $(\eta)$ for the pure forced convection case with increasing axial coordinate $(\xi)$

\section{REFERENCES}

Bég, O.A., H.S. Takhar, J. Zueco, A. Sajid and R. Bhargava (2008a). Transient Couette flow in a rotating non-Darcian porous medium parallel plate configuration: network simulation method solutions. Acta Mechanica 200, 129-144.

Bég, O.A., J. Zueco and Takhar, H.S. (2008b). Laminar free convection from a continuously-moving vertical surface in thermally-stratified non-Darcian high-porosity medium: Network numerical study. International Communications Heat Mass Transfer 35(7), 810-816.

Bég, O.A., J. Zueco and H.S. Takhar (2009). Unsteady magnetohydrodynamic Hartmann-Couette flow and heat transfer in a Darcian channel with Hall current, ionslip, viscous and Joule heating effects: Network numerical solutions. Communications in Nonlinear Science Numerical Simulation 14, 10821097.

Brown, G.M. (1960). Heat and mass transfer in a fluid in laminar flow in a circular or flat conduit. AIChemE Journal 10, 179-183.

Chen, T.S. and C.F. Yuh (1980). Combined heat and mass transfer in natural convection along a vertical cylinder. International Journal Heat Mass Transfer 23(4), 451-461.

Deen, W.M. (1998). Analysis of Transport Phenomena. Oxford University Press.

Evan, L.B., R.C. Reid and E.M. Drake (1968). Transient natural convection in a vertical cylinder. AIChemE Journal 14, 251-261.

Ganesan, P. and H.P. Rani (2000). Unsteady free convection MHD flow past a vertical cylinder with heat and mass transfer. International Journal Thermal Sciences 39, 265-272.

Gebhart, B. (1988). Buoyancy-Induced Flows and Transport. Hemisphere, Washington, USA.

Goldstein, R.J. and D.G. Briggs (1964). Transient free convection about vertical plates and circular cylinders. ASME J. Heat Transfer 86, 490-500.

Hossain, M.A. and A. Nakayama (1993). Non-Darcy free convective flow along a vertical cylinder embedded in a porous medium with surface mass flux. International Journal Heat Fluid Flow 14 (4), 385-390.
Incropera, F.P. and D.P. DeWitt (1996). Fundamentals of Heat and Mass Transfer. 4th edition, John Wiley \& Sons, New York.

Juncu, G. (2005). Conjugate heat/mass transfer from a circular cylinder with an internal heat/mass source in laminar crossflow at low Reynolds numbers. International Journal Heat Mass Transfer 48 (2), 419-424.

Kumari, M. and G. Nath (2004). Mixed convection boundary layer flow over a thin vertical cylinder with localized injection/suction and cooling/heating. International Journal Heat Mass Transfer 47 (5), 969-976.

Lira, I. (2008). Scales of free convection around a vertical cylinder. European Journal Physics 29 (6), 1301-1309.

Mamyoda, T. and K. Asano (1993). Analysis of drag coefficients and heat and mass transfer of a cylinder with diffusive surface mass suction or injection. Japanese Journal Chemical Engineering 26 (4), 382-388.

Merkin, J.H. and I. Pop (1988). A note on the free convection boundary layer on a horizontal circular cylinder with constant heat flux. Heat and Mass Transfer 22, 79-81.

Merkin, J.H. and I. Pop (1987). Mixed convection boundary-layer on a vertical cylinder embedded in a saturated porous medium. Acta Mechanica 66, 251-262.

Pspice 6.0 (1994). Irvine, California 92718. Microsim Corporation, 20 Fairbanks.

Takhar, H.S., A.J. Chamkha and G. Nath (2000). Combined heat and mass transfer along a vertical moving cylinder with a free stream. Heat and Mass Transfer 36 (3), 237-246.

Takhar, H.S., A.J. Chamkha and G. Nath (2002). Natural convection on a vertical cylinder embedded in a thermally stratified high-porosity medium. International. Journal Thermal Sciences 41 (1), 83-93.

Tien, C.L. and D.T. Campbell (1963). Heat and mass transfer from rotating cones. Journal Fluid Mechanics 17, 105-112.

Tripathi, G., K.N. Shukla and R.N. Pandey (1973). An integral equation approach to heat and mass transfer problem in an infinite cylinder. International Journal Heat Mass Transfer 16(5), 985-990.

Vincent, L. and F. Coeuret (1972). Additional data for calculations of steady heat or mass transfer in the axisymmetric boundary layer over a circular cylinder. International Journal Heat Mass Transfer 15(2), 359-360. 
J. Zueco et al. / JAFM, Vol. 4, No. 2, Issue 1, pp. 13-23, 2011.

Zueco, J., O.A. Bég, A.T. Bég and H.S. Takhar (2009). Numerical study of chemically-reactive buoyancy-driven heat and mass transfer across a horizontal cylinder in a high-porosity non-Darcian regime. Journal Porous Media 12(6), 519-535.

Zueco, J., F. Alhama and C.F. González-Fernández (2004). Analysis of laminar forced convection with network simulation in thermal entrance region of ducts. International Journal Thermal Science 43 (5), 443-451.

Zueco, J. (2006). Network method to study the transient heat transfer problem in a vertical channel with viscous dissipation. International Communications Heat Mass Transfer 33, 10791087.

Zueco, J. (2008). Unsteady free convection and mass transfer flow of viscous dissipation fluid using the electronic network simulation program SPICE: Variable properties effects. Numerical Heat Transfer Part A: Applications 53 (9), 925-944. 